\title{
The SERC 5-Year Technical Plan: Designing the Future of Systems Engineering Research
}

\author{
Jon Wade ${ }^{1(\bowtie)}$, Dinesh Verma ${ }^{1}$, Thomas McDermott ${ }^{1}$, \\ and Barry Boehm ${ }^{2}$ \\ ${ }^{1}$ Stevens Institute of Technology, \\ Castle Point on Hudson, Hoboken, NJ 07030, USA \\ \{jon.wade, dinesh. verma, tmcdermo\}@stevens.edu \\ ${ }^{2}$ University of Southern California, Los Angeles, USA \\ boehm@usc.edu
}

\begin{abstract}
The Systems Engineering Research Center (SERC), a US University Affiliated Research Center, developed the 2014-2018 Technical Plan to provide the vehicle by which to align the SERC Vision and Research Strategy with the US Federal Government Sponsor's top research priorities. This paper summarizes the SERC Vision, the Sponsor's needs, and the SERC's response to these needs. It then describes the objectives, approach and content of the original fiveyear SERC Technical Plan, and provides an overview of the results. Emerging systems challenges are noted along with the approach that is being used to address them in the upcoming Five Year Technical Plan. Finally, this paper describes the status of the new plan and some of the opportunities and challenges that it provides.
\end{abstract}

